\title{
A Survey of Mid-Air Ultrasonic Tactile Feedback
}

\author{
Ismo Rakkolainen*, Antti Sand, Roope Raisamo \\ Information Technology and Communication Sciences \\ Tampere University \\ Tampere, Finland \\ *e-mail: ismo.rakkolainen@tuni.fi
}

\begin{abstract}
This paper contains a succinct survey on the recent major advances in contactless ultrasonic tactile feedback. It is a haptic technology which enables easy mid-air interactions with rich multisensory feedback and creates effects which are otherwise impossible. It can become a disruptive technology for multimedia interfaces, applications, and mobile computing. We summarize and discuss the advantages, problems and applications of the technology. This survey provides an introduction to the topic for anyone interested in applying or researching it.
\end{abstract}

Keywords: haptics; ultrasonic tactile feedback; mid-air haptics

\section{INTRODUCTION}

Effective feedback helps users to get information, notifications and warnings. Visual or auditory feedback is often used in multimedia systems, but also mid-air ultrasound-based tactile feedback has become possible for human-computer interaction (HCI) in recent years. It has raised a lot of interest in research and commercial applications and it is employed for feedback for contactless multimedia interfaces and buttons, virtual reality (VR), touch-free interaction with cars, appliances, etc.

Ultrasound tactile actuation has two core advantages over the normal vibrotactile actuation in HCI. Firstly, it enables tactile mid-air sensations on the hand, without needing to give any visual attention to a control, or touch any device. Secondly, it can map closely to other modalities or gestures to enhance human-system interaction. Ultrasound haptics maintains the freedom of movement, is unobtrusive, and can feel magical to the user.

There are many surveys on haptics in general (e.g., [7], [58], [4]), but they all only shortly mention ultrasonic mid-air haptics. There are only two earlier surveys on the ultrasonic phased array haptics that we are aware of. One survey [14] discusses mostly experiments in $\mathrm{MHz}$ range for the purposes of physiology and medicine, and another short HCI-focused survey [2] is outdated. The technology has advanced a lot since these surveys were made. The leading company in the field, Ultrahaptics Ltd., has a knowledge base on the topic [53].

Our contribution is an up-to-date survey on mid-air ultrasound haptics. We give an overview of ultrasound haptics in Section 2 and interaction with it in Section 3. We discuss the limitations and some major advances in Section 4. We identify some key applications and use cases in Section 5. Finally, conclusions are given and future directions are discussed.

\section{HAPTIC TECHNOLOGIES}

\section{A. Haptic Devices}

Haptics is an integral part of our lives and activities. Humans use the sense of touch to grasp, explore, walk and manipulate in the real world. The sense of touch is delicately and marvelously built, it is a very complex system, and it pervades the whole body. It comprises of cutaneous inputs from the various types of mechanoreceptors in the skin and kinesthetic inputs from the muscles, tendons and joints. It provides updated information, e.g., on 3D shape and texture of objects, the position of the limbs, balance and the muscle stretch [4]. Mechanoreceptors have various densities in various body parts. The sense of touch associates a certain tactile stimulation with pressure, vibration, pain, temperature or pleasure.

Haptic display is an interface for communication between human and computer. The sense of touch must be artificially recreated, e.g., in interactive computing, virtual worlds and robot teleoperation. Mechanoreceptors in the human body are stimulated to produce expedient sensations of touch. This can enhance realism and human performance. Usually tactile feedback is provided in direct contact to skin, which seems intuitive for the sensation of touch. Some haptic devices can provide also force feedback. Many technologies can be used, e.g., tactile gloves, exoskeletons or proxy devices.

The fidelity of current tactile display technologies is very rudimentary compared to audiovisual displays or to the capabilities and complexity of human tactile sensing [4]. The shortcomings of tactile display technologies amount to several orders of magnitude. Many shortcuts and approximations must be used in order to mass-produce haptic displays for general use.

Contactless haptic feedback is possible with pressurized air jets or air vortex rings, which are simple but rough feedback methods with some inherent lag. Laser-induced thermoelastic effect or electromagnetic-based haptic interface are also possible, but they require a wearable prop on finger or on hand.

Focused ultrasonic acoustic air pressure is one contactless feedback method. Focused ultrasound for HCI tactile sensations employs typically 40 or $70 \mathrm{kHz}$ frequencies, which is just inaudible sound. Here we discuss only previous work, which is relevant for these frequencies or for the ultrasound haptics for HCI. 


\section{B. Ultrasound Haptics}

Gavrilov [13] and Iwamoto et al. [23] presented ways to invoke directly the human skin by focused, modulated $40 \mathrm{kHz}$ ultrasound. Iwamoto et al. employed a phased array of 91 ultrasound transducers. Acoustic radiation force produces small skin deformations and thus elicit the sensation of touch. The devices are often called airborne ultrasound tactile displays (AUTD).

As the hand cannot feel vibrations at $40 \mathrm{kHz}$, the emitted ultrasound is modulated to create vibrations detectable by the hand. Most ultrasound haptics systems use $\sim 200 \mathrm{~Hz}$ modulation and trigger Lamellar corpuscles, which are dense on palm, and which sense vibration and pressure. Other frequencies and mechanoreceptors elsewhere on the body can also be used, such as Meissner corpuscles on face [16], or Merkel cell disks and Ruffini corpuscles on human upper body [51]. Ultrasound haptics can even be perceived through thin clothes.

The sequence in Fig. 1 illustrates the principle of an ultrasonic phased transducer array. The array times and focuses the sound waves, and they form a focal point in the 3D space above the phased array. The focal point cannot be fully singular due to wavelength limitation of $8.5 \mathrm{~mm}$ (for $40 \mathrm{kHz}$ ), and because there are always also some grating lobes (secondary intensity peaks).
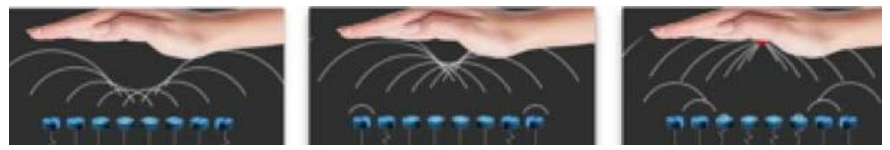

Figure 1. The principle of an ultrasonic phased array. Correctly timed ultrasound waves create acoustic pressure in the focus point [53].

The focal point movement can be controlled fast, and there can be several focal points or shapes [31]. Also haptic textures can be displayed (e.g., [37], [10]). Ultrasonic transducer arrays can be instructed with low-level commands, but easy editors (e.g., UltraHaptics' Sensation Editor) help haptic sensation designers. Fast and accurate hand tracking is also needed. Fig. 2 shows a typical system overview and a depiction of the effect.

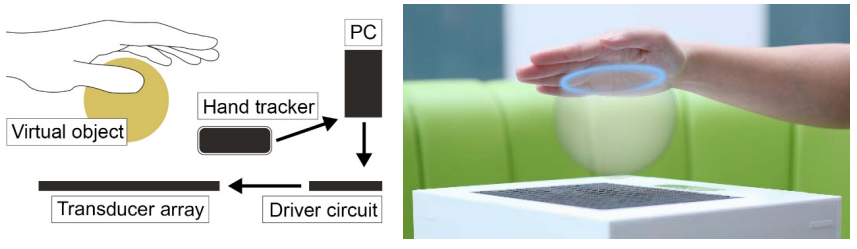

Figure 2. Left: typical system overview.

Right: a depiction of the tactile sensation in mid-air [31].

The software and hardware of commercial systems cannot be easily modified or adapted. Ultraino [33] is an open source, multiplatform phased array control system for airborne ultrasound transmission. It allows users to define array geometries and then visualize the resulting acoustic fields.

\section{Other Applications of Phased Ultrasound Arrays}

Applications of ultrasound haptics have expanded to new intriguing fields. It can, e.g., be used to move physical objects on interactive tables or to levitate ultralight particles in order to create mid-air displays. Audible, highly directed sound can be created with focused ultrasound [39]. Acoustic streaming tech- nology (Bessel beams) can be used to control narrow airflows, e.g., for fragrance distribution or removal, or to deliver a cooling sensation remotely via ultrasound-driven airflow.

\section{INTERACTION WITH ULTRASOUND HAPTICS}

Touchless interfaces based on gestures, body tracking, facial expression recognition and gaze tracking have many advantages but also a significant drawback: the lack of tactile feedback. Interaction without it may feel unreal and can lead to uncertainty. The user may wonder: "did the action register?"

Ultrasonic tactile feedback can make touchless interfaces feel more natural and reassure the user that the hand was indeed in contact with an object. Many user tests affirm that it provides easy-to-use interaction and improves the usability.

Several studies on measuring the perception of ultrasound haptics have been published. It seems to have similar properties as vibrotactile feedback on the perceptibility of frequencies. Early research focused on the detection of one [20] or multiple points of feedback [6]. The sense of touch in fingers and palms is the most sensitive to vibration of $150-250 \mathrm{~Hz}$ [46]. A good form of mid-air tactile feedback for a button click is a single $0.2 \mathrm{~s}$ burst of $200 \mathrm{~Hz}$ modulated ultrasound [40].

The minimum distance on the skin that is required to recognize the position difference between a projected visual point image and a tactile stimulation is about $10-13 \mathrm{~mm}$ regardless of the stimulation patterns [60]. The average localization error of a static point is $8.5 \mathrm{~mm}$ [59]. Stimulation of multiple points along the trajectory, longer durations (50-200 ms) and longer traveling distances $(>3 \mathrm{~cm})$ all improve movement perception. Raza et al. [44] presented a perceptually correct haptic rendering algorithm which is independent of the hardware.

Several aspects of the use and interaction of mid-air ultrasound tactile feedback have been researched. Carter et al. [6] provided multi-point haptic feedback above an interactive screen. The transducers were under a front projection screen, which allowed ultrasound to pass through it. A similar visioacoustic screen [61] allowed ultrasonic pressure to pass through it while back projecting light on screen. They also presented two interaction layers: a guide layer for hand guidance, and an operational layer near the screen for button presses etc. Mid-air haptic shapes are not easily identified even after a learning phase [45]. Linear shapes are easier to recognize than circular shapes, and increasing age decreases tactile sensitivity.
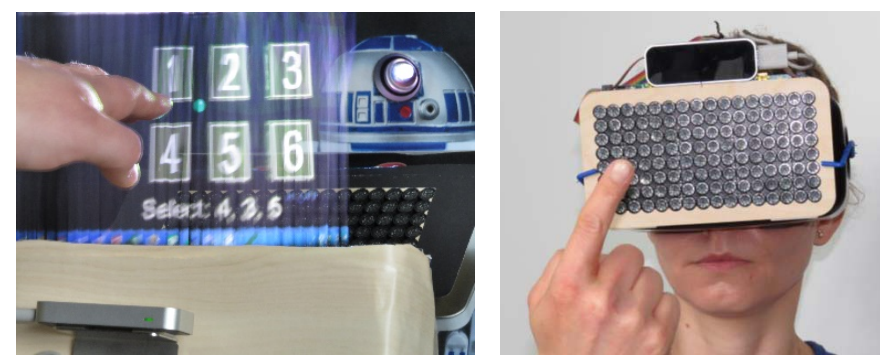

Figure 3. Left: mid-air fogscreen with mid-air haptics [47].

Right: mid-air ultrasound haptics interaction in front of an HMD [48].

Interaction with "hologram" autostereoscopic displays (e.g., [19]), a particle display floating in mid-air [47] (see Fig. 3 left) 
and a VR headset with ultrasound haptics (e.g., [48], [25]) (see Fig. 3 right) have been presented. An interactive system [34] enables a user to manipulate $3 \mathrm{D}$ object images with multiple bare fingers receiving haptic feedback. It improves the recognition of the object surface angle and position and it enables the user to hold and move the object easily even if it is not visible.

\section{LIMITATIONS}

Ultrasound haptics has some inherent limitations, which have an impact on potential applications. Below we list the major limitations and recent innovations to mitigate them.

Millimeter precision is hard or impossible to attain. $40 \mathrm{kHz}$ ultrasound has an $8.5 \mathrm{~mm}$ wavelength, and it inherently limits the precision. Also the summative acoustic field spreads a little and never forms a singular focus, and the grating lobes on the sides may confuse the user. Randomized array [13] or Fibonacci spiral array [42] can remove most of the grating lobes. In addition, a precise tracking system is crucial so that the ultrasound stimulation does not go to a wrong location.

Strength of the effect is an issue, as the mid-air tactile force is only a small fraction of the force thresholds of hardware buttons. A large number of transducers strengthen the effect, but not linearly, due to the limited directivity of the transducers [27]. Large arrays make the device bigger, heavier and more costly. Also the update rate impacts the perception [12]. Older devices used amplitude modulation (switching transducers off and on fast). Lateral or spatiotemporal modulation [52], [11] moves the focus continuously while maintaining the acoustic power at maximum, so that it feels stronger, more precise, and is not limited to discrete focal points.

Novel array hardware setups may strengthen the effect. A hybrid focus from $40 \mathrm{kHz}$ and $70 \mathrm{kHz}$ arrays produced stronger effect [22] than either alone. Acoustic metamaterials may extend the performance and range of transducer arrays [6], [35]. The arrays can be, e.g., parabolic or spherical. Inoue et al. [21] built a large octagonal-prism type phase array, where eight planar arrays faced each other. The acoustic radiation field was a spatially standing haptic image of lines, points or surfaces.

The range of the effect is limited. Close to the array (under $1 \mathrm{~cm}$ ), the effect is not possible, and far from it (tens of $\mathrm{cm}$, depending on the array size), the effect gets increasingly weak and unprecise. The placement of the array needs to be considered carefully for each application. A distributed, multiunit ultrasound phased array can reach a distance of $1 \mathrm{~m}$ [18]. If a small array is mounted on the user (e.g., on wrist [59] or on an HMD [48]), the range inherently moves with the user. Encounter-type setups (e.g., with robot arms [2]) can expand the range, but the hand tracking needs to be reliable, because even one major failure may cause a painful disaster with the robot arm.

One limitation is the current size and weight of the arrays, because a large number of piezoelectric ceramic transducers is needed. There is a trade-off between portability and feedback intensity. At least 100 transducers are needed for a good effect, depending on the desired distance, strength, arrangement, etc. If a transducer costs 1 USD, a 100-piece array would cost over 100 USD. The ultrasound array also consumes power, which is an issue especially for mobile and portable devices.
Smaller transducers are being developed. Flexible printed circuit technology could make very thin and flexible ultrasonic emitters [24], [55] and bring down the cost. They could possibly also use transparent electrodes, enabling the mid-air tactile elements to be pasted on a visual display. However, the technology is not yet ready for commercial exploitation.

A physiological limitation is that the modulated signals at $\sim 200 \mathrm{~Hz}$ can only be felt by the palm of a hand (Lamellar corpuscles). The dorsal side of hand and all the other areas of the human body are numb to it. Thus, the relative orientation between the hand and the array has an effect on the ultrasound tactile feedback. Lower $\mathrm{Hz}$ modulation can be felt in other body parts. There are also personal variances on sensitivity.

The devices may create faint audible noise. Also, microphones may cause audible noise e.g., in nearby mobile phones. Some animals can hear ultrasound signals. Dogs can hear frequencies up to $45 \mathrm{kHz}$ and cats can hear frequencies up to 79 $\mathrm{kHz}$, so ultrasound at home may have an effect on them.

Safety is an important consideration. Because ultrasound cannot be heard, there is a possibility that dangerous exposure in the strong focal point could go unnoticed. Exposure limit recommendations for continuous $40 \mathrm{kHz}$ ultrasound are $110 \mathrm{~dB}$ and peak $140 \mathrm{~dB}$ [28], [29], [50]. Recent studies [8], [29] specifically on ultrasound haptics suggest that the systems are usually safe. We estimate that even a dislocation of $10 \mathrm{~cm}$ away from the focal point would reduce the volume by $20 \mathrm{~dB}$ (1/100th of power), and user's head is typically $30-60 \mathrm{~cm}$ away from it.

\section{APPLICATIONS OF ULTRASOUND HAPTICS}

Despite the limitations, ultrasound haptics can be used for a wide range of user interaction or notification scenarios. Mid-air tactile feedback can enhance performance [57] in some tasks, whereas in some others it does not have significant efficiency impact [9]. Even then the users usually heavily prefer it.

Home applications cover a wide array of applications, e.g., guidance, confirmation, information, warning, and changing status [54]. These categories can be applied to many contexts.

Notifications are suitable types of information for mid-air tactile feedback, e.g., receiving incoming calls or messages while having a phone nearby. Also, navigation and guidance cues are possible. Interpersonal messages can be e.g., haptic icons. Some information coded with haptic patterns can be delivered to hand, and it is hidden from others nearby, but it can transfer only relatively simple information. Mid-air haptics could also be used for warnings, e.g., a parking proximity warning onto the driver's hand.

Mid-air haptics is used for advertising and digital signage with large displays. Marketing campaigns try to grasp the attention and reach customers in a compelling way. Mid-air haptics can improve the overall advertising experience while interacting with digital screens, increase the engagement of interaction, dwell time, and brand recall. It also contributes to practical issues with touchscreen signage such as responsiveness, hygiene and cleaning requirements. It can increase significantly user engagement, usability and aesthetic appeal [30]. 
E-commerce suffers from the lack of tactile information that could help the online consumer to make purchase decisions. Mid-air haptics can deliver information on material and geometric product properties.

Virtual and augmented reality try to create immersive or compelling experiences through integrating multimodal experiences, including tactile stimuli [58]. Haptic devices have been applied to synthetic worlds since 1967 [5]. Ultrasound tactile feedback is a very promising technology for VR, as it is unobtrusive, easy to use, and does not disrupt the feeling of presence, unlike other haptic devices. The illusion of falling raindrops in VR created a very believable and realistic experience [41]. Mid-air tactile feedback can be used for HMDs so that the user can touch virtual objects. If the phased array is placed to the front of an HMD [48] (see Fig. 3 right), the tactile feedback is always directed to the visual working area of the user, and its range is adequate (the convenient reach volume of an arm). If the array is in a fixed position [25], it limits the working range. Cues onto the face of an HMD user are also possible [16].

Social VR and AR, interpersonal communication, and telehaptics are also related applications. Touch is a powerful vehicle for communication between humans. In a study to explore the communication of emotions through mid-air tactile stimulation, it was found that participants were able to express and recognize emotional meanings through it [38].

There are many kinds of $3 D$ and volumetric visual displays [3]. The mid-air images match well together with mid-air haptics. Several floating image displays merged with mid-air tactile feedback have been presented [19], [36], [43], [32], [26].

Games and entertainment such as videos, movies and mobile TV can be enhanced with mid-air tactile feedback. Ablart et al. [1] found that valence and arousal increases significantly when mid-air haptics augments a short movie.

Art and product design can be enhanced with haptic effects, e.g., to augment a painting with ultrasound haptics. Vi et al. [56] designed multisensory art experiences with mid-air haptics. It made art more engaging and stimulating.

Medicine and healthcare have many application opportunities. For example, surgeons rely on medical images, but it is hard to interact with them during surgery due to the need to maintain sterility. Ultrasound haptics can create contactless feedback. Also rehabilitation and medical simulators for training and education can use it, and it can deliver, e.g., Braille dots or Morse code to visually impaired users [13].

Hygiene is sometimes of great importance, e.g., for medical uses, in food factories, public spaces, or if the hands are messy in kitchen or in garage. Ultrasound haptics delivers tactile feedback in a touch-free, hygienic manner. This is a benefit for vending machines and information kiosks during flu seasons.

Cars increasingly employ touchscreens for all adjustments, but the tactile feedback of traditional buttons and knobs is lost. Touchscreen controls can reduce road safety as the driver is often required to shift focus between the road and the screen. Speech and audio feedback are not very effective replacements and they interfere with other audio signals and external noise [15]. Ultrasound haptic feedback helps a driver to feel the con- trol status in mid-air. This can reduce visual demand, shorten interaction times, improve accuracy, minimize eyes-off-theroad time on displays, and thus improve safety [49], [17], [15].

Most of the abovementioned applications are for locationbased setups and not portable. We assume that various mobile computing applications are viable use cases for ultrasound tactile feedback. For example, it could enhance ordinary, foldable, or autostereoscopic mobile phone screens, or help on using mobile phones with tactile and invisible feedback.

\section{CONCLUSIONS}

In this paper, we summarized the state of the art in mid-air ultrasonic tactile feedback, its limitations and applications. It can feel magical to the user who can have a tactile sensation in mid-air with bare hand. Among its challenges are, e.g., the range and strength of the effect, strongest effect on palm, device weight, size and price. There has been many technical advances, and ultrasound haptics is increasingly suitable for many tasks and applications.

Ultrasound haptics is still little-explored design space. It is important to conduct rigorous and controlled research to understand the technical, perceptual, and experiential limits and possibilities afforded by it. There is also hope for breakthrough technical advances, such as flexible printed circuit ultrasonic emitters and acoustic metamaterials.

\section{REFERENCES}

[1] D. Ablart, C. Velasco, and M. Obrist, "Integrating mid-air haptics into movie experiences," in Proc. ACM Int. Conf. on Interactive Experiences for TV and Online Video (TVX 2017), pp. 77-84, 2017.

[2] F. Arafsha, L. Zhang, H. Dong, and A. El Saddik, "Contactless Haptic Feedback: State of the Art," in Proc. IEEE Int. Symposium on Haptic, Audio and Visual Environments and Games (HAVE'15), pp. 1-6, 2015.

[3] P. Benzie, J. Watson, J. Surman, I. Rakkolainen, K. Hopf, H. Urey, V. Sainov, and C. von Kopylow, "A survey of 3DTV displays: techniques and technologies," IEEE T. Circ. Syst. Vid. 17, 11, pp. 1647-1658, 2007.

[4] S. Biswas and Y. Visell, "Emerging Material Technologies for Haptics," Advanced Materials Technologies 4, 4. WILEY, 2019.

[5] F. Brooks, M. Ouh-Young, J. Batter, and J. Kilpatrick, "Project GROPE - Haptic displays for scientific visualization," in SIGGRAPH Comput. Graph. 24, 4, pp. 177-185, 1990.

[6] T. Carter, S. Seah, B. Long, B. Drinkwater, S. Subramanian, "UltraHaptics: multi-point mid-air haptic feedback for touch surfaces," in $A C M$ Symp. User interface software \& tech. (UIST'13), pp. 505-514, 2013.

[7] H. Culbertson, S. Schorr, and A. Okamura, "Haptics: The Present and Future of Artificial Touch Sensation," Annual Review of Control, Robotics, and Autonomous Systems 1:385-409, 2018.

[8] A. Di Battista, "The effect of $40 \mathrm{kHz}$ ultrasonic noise exposure on human hearing", in Proc. 23rd Int. Cong. Acoustics, pp. 4783-4788, 2019.

[9] E. Freeman, S. Brewster, and V. Lantz, "Tactile Feedback for AboveDevice Gesture Interfaces: Adding Touch to Touchless Interactions," in Proc. Int. Conf. Multimodal Interaction (ICMI'14), pp. 419-426, 2014.

[10] E. Freeman, R. Anderson, J. Williamson, G. Wilson, and S. Brewster, "Textured surfaces for ultrasound haptic displays," in Proc. 19th ACM Int. Conf. Multimodal Interaction (ICMI'17), pp. 491-492, 2017.

[11] W. Frier, D. Ablart, J. Chilles, B. Long, M. Giordano, M. Obrist, and S. Subramanian, "Using Spatiotemporal Modulation to Draw Tactile Patterns in Mid-Air," in Haptics: Science, Technology, and Applications, vol. 10894, Springer, pp. 270-281, 2018.

[12] W. Frier, D. Pittera, D. Ablart, M. Obrist, and S. Subramanian, "Sampling strategy for ultrasonic mid-air haptics," in Proc. ACM CHI Conf. Human Factors in Computing Systems (CHI 2019), Paper 121, 2019. 
[13] L. Gavrilov, "The possibility of generating focal regions of complex configurations in application to the problems of stimulation of human receptor structures by focused ultrasound," Acoust. Phys. 54, 2, 2008.

[14] L. Gavrilov, E. Tsirulnikov, "Focused ultrasound as a tool to input sensory information to humans (Review)," Acoust. Phys. 58, 1, 2012.

[15] O. Georgiou, V. Biscione, A. Harwood, D. Griffiths, M. Giordano, B. Long, and T. Carter, "Haptic In-Vehicle Gesture Controls," in Proc. 9th Int. Conf. on Automotive User Interfaces and Interactive Vehicular Applications Adjunct (AutomotiveUI'17), pp. 233-238, 2017.

[16] H. Gil, H. Son, J. Kim, and I. Oakley, "Whiskers: Exploring the Use of Ultrasonic Haptic Cues on the Face," in Proc. CHI Conf. on Human Factors in Computing Systems (CHI '18). paper 658, 2018.

[17] K. Harrington, D. Large, G. Burnett, O. Georgiou, "Exploring the use of mid-air ultrasonic feedback to enhance automotive user interfaces," in Proc. ACM AutomotiveUI'18, pp. 11-20, 2018.

[18] K. Hasegawa, H. Shinoda, "Aerial Vibrotactile Display Based on Multiunit Ultrasound Phased Array," IEEE T. Haptics, 11, 3, 367-377, 2018.

[19] T. Hoshi, D. Abe, H. Shinoda, "Adding tactile reaction to hologram," in IEEE Robot \& Human Interact. Communic. (ROMAN), pp. 7-11, 2009.

[20] T. Hoshi, M. Takahashi, T. Iwamoto, and H. Shinoda, "Noncontact Tactile Display Based on Radiation Pressure of Airborne Ultrasound," IEEE Transactions on Haptics, 3 (3), pp. 155-165, 2010.

[21] S. Inoue, Y. Makino, and H. Shinoda, "Active touch perception produced by airborne ultrasonic haptic hologram," in Proc. IEEE World Haptics Conference (WHC'15), pp. 362-367, 2015.

[22] M. Ito, D. Wakuda, Y. Makino, and H. Shinoda, "Hybrid Focus Using 70 and $40 \mathrm{kHz}$ Ultrasound in Mid-Air Tactile Display," in Haptic Interaction, vol. 432. Springer, pp. 131-134, 2016.

[23] T. Iwamoto, M. Tatezono, and H. Shinoda, "Non-contact Method for Producing Tactile Sensation Using Airborne Ultrasound," in Haptics: Perception, Devices and Scenarios, pp. 504-513. Springer, 2008.

[24] T. Kamigaki, A. Noda, H. Shinoda, "Thin and flexible airborne ultrasound phased array for tactile display," in SICE'17, pp. 736-739, 2017.

[25] C. Kervegant, F. Raymond, D. Graeff, J. Castet, "Touch hologram in mid-air," in ACM SIGGRAPH 2017 Emerg. Tech. Article 23, 2017.

[26] Y. Kimura, Y. Makino, H. Shinoda, "Computer-created interactive 3D image with midair haptic feedback," in Haptic Interaction, v. 432, 2018.

[27] G. Korres and M. Eid, "Haptogram: Ultrasonic Point-Cloud Tactile Stimulation," IEEE Access, vol. 4, pp. 7758-7769, 2016.

[28] M. Lenhardt, "Airborne ultrasonic standards for hearing protection," in Proc. 9th Int. Congress on Noise as a Public Health Problem, 2008.

[29] M. Liebler, C. Kling, B. Best, A. Gerlach, and C. Koch, "Quantitative characterization of high-intensity focused airborne ultrasonic fields", in Proc. 23rd Int. Cong. Acoustics, pp. 6338-6345, 2019.

[30] H. Limerick, R. Hayden, D. Beattie, O. Georgiou, and J. Müller, "User engagement for mid-air haptic interactions with digital signage," in Proc. ACM Int. Symp. Pervasive Displays (PerDis'19), Article 15, 2019.

[31] B Long, S Seah, T Carter, S Subramanian, "Rendering volumetric haptic shapes in mid-air using ultrasound," ACM Trans. Graph. 33, 6, art. 181.

[32] Y. Makino, Y. Furuyama, S. Inoue, H. Shinoda, "HaptoClone (hapticoptical clone) for mutual tele-environment by real-time $3 \mathrm{~d}$ image transfer with midair force feedback," in $A C M$ CHI'16, pp. 1980-1990. 2016.

[33] A. Marzo, T. Corkett, and B. Drinkwater, "Ultraino: An Open PhasedArray System for Narrowband Airborne Ultrasound Transmission," IEEE Trans. Ultrason. Ferr., 65, 1, pp. 102-111, 2018.

[34] A. Matsubayashi, Y. Makino, and H. Shinoda, "Direct Finger Manipulation of 3D Object Image with Ultrasound Haptic Feedback," in Proc. CHI Conf. Human Factors in Comput. Syst. (CHI'19), Paper 87. 2019.

[35] G. Memoli, M. Caleap, M. Asakawa, D. Sahoo, B. Drinkwater, and S. Subramanian, "Metamaterial bricks and quantization of meta-surfaces," Nature Communications vol. 8, Article 14608. 2017.

[36] Y. Monnai, K. Hasegawa, M. Fujiwara, K. Yoshino, S. Inoue, and H. Shinoda, "HaptoMime: mid-air haptic interaction with a floating virtual screen," in Proc. ACM UIST'2014, pp. 663-667, 2014.

[37] Y. Monnai, K. Hasegawa, M. Fujiwara, K. Yoshino, S. Inoue, and H. Shinoda, "Adding Texture to Aerial Images Using Ultrasounds," in Haptic Interaction, vol 277. Springer, 2015.
[38] M. Obrist, S. Subramanian, E. Gatti, B. Long, and T. Carter, "Emotions Mediated Through Mid-Air Haptics," in Proc. 33rd ACM Conf. on Human Factors in Computing Systems (CHI'15), pp. 2053-2062, 2015.

[39] Y. Ochiai, T. Hoshi, and I. Suzuki, "Holographic whisper: rendering audible sound spots in three-dimensional space by focusing ultrasonic waves," in Proc. ACM CHI'17, pp. 4314-4325, 2017.

[40] K. Palovuori, I. Rakkolainen, A. Sand, "Bidirectional Touch Interaction for Immaterial Displays," in Academic MindTrek Conf. 2014, pp. 76-78.

[41] D. Pittera, E. Gatti, and M. Obrist, "I'm Sensing in the Rain: Spatial Incongruity in Visual-Tactile Mid-Air Stimulation Can Elicit Ownership in VR Users," in Proc. ACM CHI 2019, paper 132, 2019.

[42] A. Price, B. Long, "Fibonacci spiral arranged ultrasound phased array for mid-air haptics," IEEE Int. Ultrasonics Symp. (IUS), pp. 1-4, 2018.

[43] I. Rakkolainen, A. Sand, and K. Palovuori, "Mid-air user interfaces employing particle screens," IEEE Comput. Graph. 35, 2, pp. 96-102, 2015.

[44] A. Raza, W. Hassan, T. Ogay, I. Hwang, and S. Jeon, "Perceptually Correct Haptic Rendering in Mid-Air using Ultrasound Phased Array," in Proc. IEEE Transactions on Industrial Electronics, 2019.

[45] I. Rutten, W. Frier, L. van den Bogaert, and D. Geerts, "Invisible Touch: How Identifiable are Mid-Air Haptic Shapes?," in Proc. Ext. Abs. CHI Human Factors in Comput. Syst. (CHI EA'19), paper LBW0283, 2019.

[46] J. Ryu, J. Jung, G. Park, S. Choi, "Psychophysical model for vibrotactile rendering in mobile device," Presence Teleop. Virt. 19,4, 364-387, 2010.

[47] A. Sand, I. Rakkolainen, P. Isokoski, R. Raisamo, K. Palovuori, "Lightweight Immaterial Particle Displays with Mid-Air Tactile Feedback," in IEEE Haptic Audio-Visual Environments \& Games' 15, pp. 30-34, 2015.

[48] A. Sand, I. Rakkolainen, P. Isokoski, J. Kangas, R. Raisamo, K. Palovuori, "Head-mounted display with mid-air tactile feedback," in $A C M$ Symp. Virtual Reality Software and Technology (VRST'15), pp. 51-58, 2015.

[49] G. Shakeri, J. Williamson, and S. Brewster, "May the Force Be with You: Ultrasound Haptic Feedback for Mid-Air Gesture Interaction in Cars," in Proc. ACM AutomotiveUI'2018, pp. 1-10. 2018.

[50] B. Smagowska and M. Pawlaczyk-Łuszczyńska, "Effects of Ultrasonic Noise on the Human Body - A Bibliographic Review," Int. J. Occupational Safety and Ergonomics, 19:2, pp. 195-202, 2015.

[51] S. Suzuki, R. Takahashi, M. Nakajima, K. Hasegawa, Y. Makino, and H. Shinoda, "Midair Haptic Display to Human Upper Body," in Proc. Soc. Instrument and Control Engineers Japan (SICE'18), pp. 848-853, 2018.

[52] R. Takahashi, K. Hasegawa, and H. Shinoda, "Lateral Modulation of Midair Ultrasound Focus for Intensified Vibrotactile Stimuli," in Haptics: Science, Technology and Applications, pp. 276-288. Springer, 2018.

[53] Ultrahaptics Ltd. Knowledge base. (October 2019). [online]. Available: https://developer.ultrahaptics.com/knowledge-base/.

[54] L. van den Bogaert, D. Geerts, and I. Rutten, "Grasping the Future: Identifying Potential Applications for Mid-Air Haptics in the Home," in Proc. CHI '2019 Ext. Abstracts, Paper LBW1415, 2019.

[55] P. van Neer, A. Volker, A. Berkhoff, H. Akkerman, T. Schrama, A. van Breemen, G. Gelinck, "Feasiblity of Using Printed Polymer Transducers for Mid-Air Haptic Feedback," in IEEE Int. Ultrasonics Symp. (IUS '2018), pp. 1-4, 2018.

[56] C. Vi, D. Ablart, E. Gatti, C. Velasco, and M. Obrist, "Not just seeing, but also feeling art: Mid-air haptic experiences integrated in a multisensory art exhibition," Int. J. Human-Comput. Studies 108, pp. 1-14. 2017.

[57] D. Vo, S. Brewster, "Touching the Invisible: Localizing Ultrasonic Haptic Cues," in IEEE World Haptics Conf. (WHC'15), pp. 368-373, 2015.

[58] D. Wang, Y. Guo, S. Liu, Y. Zhang, W. Xu, and J. Xiao, "Haptic display for virtual reality: progress and challenges," Virtual Reality \& Intelligent Hardware, 1, 2, pp. 136-162, 2019.

[59] G. Wilson, T. Carter, S. Subramanian, and S. Brewster, "Perception of Ultrasonic Haptic Feedback on the Hand: Localisation and Apparent Motion," in Proc. ACM CHI'14, pp. 1133-1142, 2014.

[60] K. Yoshino, K. Hasegawa, H. Shinoda, "Measuring Visio-Tactile threshold for Visio-Tactile Projector,” Proc. SICE'12, pp. 1996-2000, 2012.

[61] K. Yoshino and H. Shinoda, "Visio-acoustic screen for contactless touch interface with tactile sensation," in Proc. World Haptics Conf. (WHC '13), pp. 419-423, 2013. 